Disclosure of Interests: Yilmaz Ozyazgan Speakers bureau: ABBVIE, Didar Ucar: None declared, Mustafa Erdogan: None declared, Yesim Ozguler: None declared, Gulen Hatemi Consultant for: Abbvie, Amgen, BMS, Janssen, MSD, Pfizer, UCB, Speakers bureau: Abbvie, Amgen, BMS, Jansen, MSD, Pfizer, UCB, Sebahattin Yurdakul: None declared, Vedat Hamuryudan Consultant for: Abbvie, Amgen, BMS, Jansen, MSD, Pfizer, UCB, Speakers bureau: Abbvie, Amgen, BMS, Jansen, MSD, Pfizer, UCB, Izzet Fresko: None declared, Melike Melikoglu: None declared, Emire Seyahi: None declared, Serdal Ugurlu: None declared, Hasan Yazici: None declared

DOI: 10.1136/annrheumdis-2019-eular.4317

\section{SAT0224 PRESENTATION, MANAGEMENT AND OUTCOME OF ANCA ASSOCIATED VASCULITIDES IN ITALY: A 20- YEAR FOLLOW-UP STUDY}

Mara Felicetti, Roberto Padoan, Mariagrazia Lorenzin, Augusta Ortolan, Franco Schiavon. Rheumatology, Unit, University of Padova, Italy, Department of Medicine DIMED, Padova, Italy

Background: ANCA associated vasculitis (AAV) are systemic diseases with a wide spectrum of clinical presentation and organ involvement.

Objectives: To analyze presentation, management and 5 years-outcomes of AAV patients diagnosed between 2000 and 2018 in the monocentric cohort of Padova Vasculitis Center.

Methods: We retrospectively collected all AAV patients diagnosed between 2000 and 2018 and followed in the Vasculitis Center of Padova University. We focused on demographic and clinical features at baseline, first line immunosuppressive (IS) treatment, 6 months mortality, 5 years-relapse rate and survival rate and. In the analysis, we kept separate eosinophilic granulomatosis with polyangiitis (EGPA) from granulomatosis with polyangiitis (GPA) and microscopic polyangiitis (MPA).

Results: We identified 171 patients (F/M 93/78) with AAV diagnosed since 2000 (61 between 2000-2010 and 110 between 2011-2018,\% of increased in diagnosis rate $+183 \%)$ : $82(48 \%)$ were GPA, $28(16 \%)$ MPA and $61(36 \%)$ EGPA. The patients were mostly Caucasian $(98 \%)$, with a mean age at diagnosis of $58.4 \pm 16.1$ years, slightly younger if diagnosed in the second decade (2010-2018) than in first decade (2000-2010) (59.5 \pm 16.6 years vs $61.6 \pm 14.7$ years, $p=0.049$ ). ANCA were tested in 163 patients: $23 \%$ ANCA negative, 37\% cANCA/PR3, 39\% pANCA/MPO and $1 \%$ double positive.

GPA/MPA patients were mostly ANCA positive at diagnosis (96/110 patients), in particular we counted 12 (15\%) GPA-ANCA negative patients, 55 (68\%) GPA-PR3, 13 (16\%) GPA-MPO, 1 GPA with double positivity while MPA resulted only ANCA positive, 22 (82\%) MPA-MPO and 5 (19\%) MPA-PR3. In EGPA group, only 28/54 presented with ANCA positivity, all $\mathrm{p}-\mathrm{ANCA} / \mathrm{MPO}$ specificity.

Disease severity at diagnosis was assessed only in GPA/MPA patients: 12/101 limited disease, 34 early systemic, 29 generalized and 26 severe. In particular, GPA/MPA more frequently presented with systemic symptoms (76\%), ENT and lung involvement (respectively $62 \%$ and $66 \%$ ). Renal vasculitis was reported in $65 \%$ of patients with a mean eGFR of $50.2 \pm 39.8 \mathrm{ml} / \mathrm{min}$. Moreover, we registered 17 alveolar haemorrhages, 4 cardiac involvement and 3 gastrointestinal (GI) disease.

EGPA patients, instead, presented more frequently with ENT (85\%) and lung involvement (96\%) (mostly uncontrolled asthma and pulmonary infiltrates). $31(54 \%)$ EGPA presented nerve involvement (4 with CNS involvement) and $9(16 \%)$ with cardiac involvement. Interestingly, no renal or Gl vasculitis was reported.

The first line IS treatment administered was: cyclophosphamide (CYC) in $66(38 \%)$, rituximab (RTX) in $14(8 \%)$, azathioprine (AZA) in $27(16 \%)$, methotrexate (MTX) in $25(14 \%)$ and mycophenolate (MMF) in only 5 $(3 \%)$.

Mortality rate at 6 months in all cohort resulted of $1.2 \%$ (2 event), while the 5-years survival calculated with Kaplan Meier method was 94.3\% (8 events). All deaths occurred in the GPA/MPA group. A relapse occurred in $36 \%$ of patients, with a significant higher frequency in GPA/MPA than in EGPA (46.3\% vs $17 \%, p=0.017)$.

Conclusion: Our cohort is characterized by significant higher rate of AAV diagnosis in the last decade and this could reflect the increasing incidence and prevalence reported in literature ${ }^{1}$. Interestingly, GPA diagnosis, especially with cANCA/PR3 specificity, was prevalent in our cohort despite some authors reported an higher prevalence of MPA in Mediterrean area $^{1-2}$. Finally, EGPA confirmed a better outcome at last follow up than GPA and MPA, but we noted that no renal and $\mathrm{Gl}$ involvement was observed in our EGPA patients.

\section{REFERENCE}

[1] Berti A, Dejaco C. Update on the epidemiology, risk factors, and outcomes of systemic vasculitides. Best Pract Res Clin Rheumatol. 2018;32(2):271 294.

[2] Romero-Gómez C, et al. Epidemiological study of primary systemic vasculitides among adults in southern Spain and review of the main epidemiological studies. Clin Exp Rheumatol. 2015;33(2 Suppl 89):S-11-8

Disclosure of Interests: None declared

DOI: 10.1136/annrheumdis-2019-eular.5112

\section{SAT0225 TARGETED SERUM METABOLIC PROFILE IN PATIENTS WITH TAKAYASU ARTERITIS}

Dinesh Kumar ${ }^{1}$, Harikrishnan Gangadharan Nair ${ }^{2}$, Avinash Jain ${ }^{3}$,

Anupama Guleria ${ }^{1}$, Umesh Kumar ${ }^{1}$, Durga Misra ${ }^{3}$, Ramnath Misra ${ }^{3} .{ }^{1} C B M R$,

Lucknow, India; ${ }^{2}$ Sanjay Gandhi Post graduate Institute of Medical Sciences,

Clinical immunology, Lucknow, India; ${ }^{3}$ Sanjay Gandhi Post graduate Institute of

Medical Sciences, Clinical immunolgy, Lucknow, India

Background: Takayasu arteritis (TAK) is a chronic inflammatory arteritis that mainly affects aorta and, its major branches. Often the course is unpredictable and it remains a challenge to assess disease activity despite the availability of acute phase reactants and imaging modalities [1]. Management heavily relies on physician global assessment. Analysis of serum metabolites using nuclear magnetic resonance spectroscopy is a promising exploratory approach towards identifying new biomarkers in TAK which may cast some light on disease pathogenesis and disease activity.

Objectives: Aim of the study was to evaluate the performance of serum amino acids as determinants of disease activity in TAK.

Methods: Patients with TAK fulfilling ACR 1990 criteria were enrolled and disease activity assessed using ITAS2010 and ITAS-A[2]. Samples were analysed using 1D $1 \mathrm{H} 800 \mathrm{MHz}$ Nuclear magnetic resonance spectrometer equipped with a Cryoprobe (at $300 \mathrm{~K}$ ). The spectra were processed and the concentration of amino acids were measured with respect to internal reference metabolite formate assuming its concentration $10 \mu \mathrm{M}$ in commercial software program CHENOMX (www.chenomx.com) and the resulted normalized values were compared using Tukey's multiple comparison test and diagnostic potential was evaluated using receiver operating characteristic (ROC) curve analysis.

Results: 45 active TAK patients with ITAS-A $\geq 4$ and 53 inactive TA patients with median age 27 years [IQR, 22-35 and IQR, 23-37 years respectively] were enrolled. Female to male (F:M) ratio was $3.5: 1$ and 4.9:1 and median duration of illness 5 [IQR, 2-9 years] and 3 [IQR, 1-6 years] years in active and inactive group respectively. Majority had class $\mathrm{V}$ disease. 43 individuals served as health control $(\mathrm{HC})$ with mean age 30 years and F:M ratio of 4.3:1. Serum levels of Phenylalanine (Phe), Tyrosine (Tyr), Histidine (His), Valine (Val) and Alanine (Ala) were found to be decreased in TAK patients suggesting augmented utilization in the immune-metabolic pathways mainly to replenish the energy demand and self-repair mechanism under conditions of inflammation and oxidative stress. An analysis of amino acids ratio revealed significantly higher His/ Tyr, Ala/Tyr and Val/Tyr ratio in $\mathrm{HC}$ when compared to active TAK patients. Ratio were lower in active TAK when compared to inactive TAK patients but only $\mathrm{Val} / \mathrm{Tyr}$ ratio was significant (AUROC, 0.64 and $p$, 0.01). Ratio served as a gradient with lowest value in active disease and gradually increasing and moving towards $\mathrm{HC}$ as disease was getting controlled
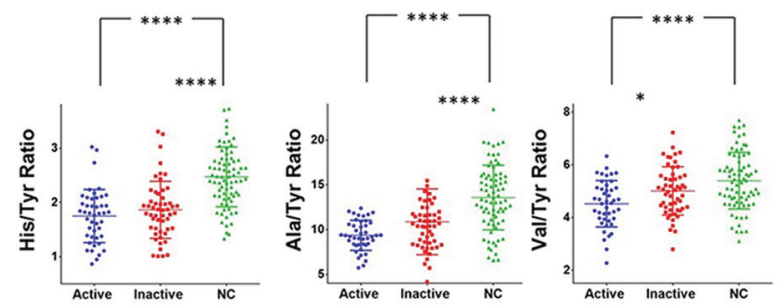

Figure 1: Comparison of various amino acid ratio in active and inactive TAK patient with respect to healthy control. Ratio served as a gradient with lowest in active disease and gradually moving towards $\mathrm{HC}$ as disease was getting controlled.

*significant $\mathrm{p}$ value; Ala, alanine; His, Histidine; NC, Normal healthy control;

Tyr, tyrosine; Val, Valine 
Conclusion: The present targeted NMR based metabolomics study demonstrated that the serum metabolic profiles of amino acid may help guide therapy and ratio of $\mathrm{Val} / \mathrm{Tyr}$ can differentiate active from inactive patients. However, future studies on large patient cohorts are required to establish its clinical utility.

\section{REFERENCES}

[1] Nakagomi D, Jayne D. Outcome assessment in Takayasu arteritis. Rheumatol. (United Kingdom) 2016;55:1159-71.

[2] Misra R, Danda D, Rajappa SM, Ghosh A, Gupta R, Mahendranath KM, et al. Development and initial validation of the Indian Takayasu Clinical Activity Score (ITAS2010). Rheumatol. (United Kingdom) 2013;52:1795-801.

Disclosure of Interests: None declared

DOI: 10.1136/annrheumdis-2019-eular.7672

\section{SAT0226 INFLIXIMAB FOR THE TREATMENT OF REFRACTORY POLYARTERITIS NODOSA}

Shira Ginsberg ${ }^{1}$, Itzhak Rosner ${ }^{1}$, Gleb Slobodin ${ }^{1}$, Michael Rosenbaum ${ }^{1}$, Lisa Kaly ${ }^{1}$, Nizar Jiries ${ }^{1}$, Nina Boulman ${ }^{1}$, Abid Awisat ${ }^{1}$, Haya Husseuin ${ }^{1}$, Irina Novofastovski ${ }^{2}$, Amal Silawy ${ }^{1}$, Doron Rimar ${ }^{1} .{ }^{1}$ Bnai-zion medical center, Haifa, Israel; ${ }^{2}$ Haemek medical center, Afula, Israel

Background: Polyarteritis Nodosa (PAN) is a necrotizing vasculitis predominantly affecting medium and small size arteries (1). Cyclophosphamide, a drug with narrow therapeutic range and poor safety profile, constitutes the treatment of choice for PAN vasculitis with major organ involvement. (2)

Objectives: To describe our clinical experience in treating refractory PAN with infliximab (a TNF inhibitor), a drug with good tolerability and better safety profile than cyclophosphamide.

Methods: Twenty-six PAN patients were admitted to our rheumatology unit between 2006 and 2017, of whom 9 patients, with severe and refractory disease, were treated with infliximab after failure of standard treatment.

We describe herein the patients' characteristics, clinical manifestations, severity and response to infliximab treatment and review the current literature.

Results: Complete remission was defined as the absence of features of active disease and withdrawal of prednisone therapy. Significant improvement was defined as clinical improvement and prednisone dose reduction of at least $50 \%$ or a $50 \%$ reduction in immune modulatory medications other than prednisone.

After 4 months of treatment, $8 / 9$ (89\%) patients achieved significant improvement, with two of them achieving complete remission.

Conclusion: We suggest anti TNF agents, and in particular infliximab, are relatively safe and efficacious treatment options in refractory PAN $A$ randomized controlled trial should be done in order to objectively evaluate infliximab in PAN.

\section{REFERENCES}

[1] Jennette JC, Falk RJ, Bacon PA, Basu N, Cid MC, Ferrario F, et al. 2012 revised International Chapel Hill Consensus Conference Nomenclature of Vasculitides. Arthritis Rheum. 2013; 65:1-11.

[2] De Virgilio A, Greco A, Magliulo G, Gallo A, Ruoppolo G, Conte M, Martellucci $S$, de Vincentiis M. Polyarteritis nodosa: A contemporary overview. Autoimmun Rev. 2016; 15:564-70.

Disclosure of Interests: None declared

DOI: 10.1136/annrheumdis-2019-eular.342

\section{SAT0227 MYOCARDIAL FIBROSIS IS ASSOCIATED WITH ANCA STATUS, ORGAN INVOLVEMENT AND DISEASE SEVERITY IN PATIENTSWITH GRANULOMATOSIS WITH POLYANGIITIS}

Alessandro Giollo ${ }^{1,2,3}$, Raluca-Bianca Dumitru ${ }^{2,3}$, Peter Swoboda ${ }^{4}$, Sven Plein ${ }^{4}$, John Greenwood ${ }^{4}$, Maya Buch ${ }^{2,3}$, Jacqueline Andrews ${ }^{3} .{ }^{1}$ Rheumatology Unit, Department of Medicine, University of Verona, Verona, Italy; ${ }^{2}$ Leeds Institute of Rheumatic and Musculoskeletal Medicine, University of Leeds, Leeds, United Kingdom; ${ }^{3}$ NIHR Leeds Biomedical Research Centre and Clinical Research Facility, Leeds Teaching Hospitals, Leeds, United Kingdom; ${ }^{4}$ Multidisciplinary Cardiovascular Research Centre, Leeds Institute of Cardiovascular and Metabolic Medicine, University of Leeds, Leeds, United Kingdom

Background: Granulomatosis with polyangiitis (GPA) is a systemic vasculitis with an increased burden of cardiovascular (CV) events compared to the general population, but the prevalence of primary cardiac involvement in this disease is unknown.

Objectives: The objective of this prospective study was to describe with cardiac magnetic resonance (CMR) the myocardial abnormalities associated with GPA and their correlations with disease characteristics.

Methods: Twenty-six patients with GPA and no prior CV disease (CVD) or diabetes mellitus underwent contrast-enhanced CMR, including late gadolinium-enhancement (LGE), T1 mapping for native T1 and extra-cellular volume (ECV) quantification for assessment of myocardial fibrosis and tissue tagging for assessment of left ventricular (LV) function. Characteris tics of disease, treatments and CV risk factors were collected; disease relapse was defined as reappearance or worsening of vasculitis symptoms requiring an increase of the current treatment or introduction of additional immunosuppressive medication. Twenty-five healthy volunteers (HV) with comparable age, sex, BMI and arterial blood pressure served as controls.

Results: Patients with GPA (median age 58 years, disease duration 8 years, BVAS 2; females $46 \%$, PR3 ANCA 58\%) had similar cardiovascular risk profile to HV. A focal, non-ischemic LGE pattern of fibrosis was detected in $24 \%$ of patients and no controls $(p=0.010$; Table 1). Patients with myocardial LGE were more frequently PR3 ANCA negative (93\% vs $7 \%, \mathrm{p}=0.007$ ), and they presented more frequently with involvement of the lower respiratory tract $(75 \%$ vs $25 \%, p=0.097)$ and skin $(63 \%$ vs $38 \%, p=0.087)$. Values of LGE-related fibrotic mass were higher in patients presenting with renal involvement $(p=0.036)$. Native T1 and ECV were higher in patients with GPA than HV (Table 1); ECV was higher in those with relapsing disease, and native $\mathrm{T} 1$ was inversely associated with PR3 ANCA $(\beta=-0.664, p=0.001)$. Peak systolic strain was slightly reduced in GPA than controls (Table 1); LV ejection function (LVEF) was inversely correlated with disease duration $(\beta=-0.454, p=0.026)$

Table 1. CMR measures $\dagger$

\begin{tabular}{lccc}
\hline LV volumes and mass & & \\
\hline LV end diastolic volume index, $\mathrm{mL} / \mathrm{m}^{2}$ & $69(61,84)$ & $79(70,89)$ & 0.105 \\
\hline LV end systolic volume index, $\mathrm{mL} / \mathrm{m}^{2}$ & $28(23,37)$ & $32(28,39)$ & 0.122 \\
LV mass index, $\mathrm{g} / \mathrm{m}^{2}$ & $37(42,47)$ & $50(42,56)$ & 0.014 \\
LV mass/LV end diastolic volume, g/ & $0.59(0.48,0.66)$ & $0.59(0.54,0.66)$ & 0.486 \\
$\mathrm{~mL}$ & & & \\
LV function & $59(56,63)$ & $58(53,62)$ & 0.351 \\
LV ejection fraction,\% & & \\
LV peak systolic strain & $-0.19(-0.21,-$ & $-0.21(-0.22,-$ & 0.017 \\
Base & $0.16)$ & $0.19)$ & \\
& $-0.21(-0.23,-$ & $-0.22(-0.23,-$ & 0.298 \\
Mid & $0.20)$ & $0.20)$ & \\
& $-0.21(-0.23,-$ & $-0.23(-0.25,-$ & 0.101 \\
Apex & $0.16)$ & $0.20)$ & \\
& $12.3(9.2,14.5)$ & $13.8(11.6,16.0)$ & 0.086 \\
Torsion, deg & $14.1(9.0,15.7)$ & $14.1(11.7,17.7)$ & 0.222 \\
Peak twist & & & \\
Myocardial tissue characterization & $1225(1199,1255)$ & $1203(1185,1233)$ & 0.006 \\
Native T1 (ms) & $25.0(23.3,27.2)$ & $23.6(20.5,25.1)$ & 0.009 \\
Extracellular volume,\% & $6 / 25(24)$ & $0 / 25(0)$ & 0.010 \\
LGE, $n$ (\%) & $1.25(0.81,2.63)$ & $0(0,0)$ & NA \\
\hline LGE scar tissue, g & &
\end{tabular}

$\uparrow$ Continuous data are presented as median (interquartile range).

Conclusion: Patients with GPA had significant myocardial abnormalities on CMR compared to HV. ANCA status, systemic organ involvement and disease severity were associated with CMR markers of myocardial fibrosis. CMR could be a useful tool for identification and future risk stratification of myocardial involvement of GPA.

Disclosure of Interests: : Alessandro Giollo: None declared, Raluca-Bianca Dumitru: None declared, Peter Swoboda: None declared, Sven Plein None declared, John Greenwood: None declared, Maya Buch Grant/ research support from: Pfizer LTD, UCB, Consultant for: AbbVie, Eli Lilly, EMD Serono, Pfizer Ltd., Sanofi, Jacqueline Andrews: None declared DOI: 10.1136/annrheumdis-2019-eular.4966

\section{SAT0228 LEUKOCYTE DYNAMICS IN GIANT CELL ARTERITIS AND POLYMYALGIA RHEUMATICA PATIENTS BEFORE, DURING AND AFTER TREATMENT}

Jacoba Graver, Yannick van Sleen, Wayel Abdulahad, Kornelis van der Geest, Annemieke Boots, Maria Sandovici, Elisabeth Brouwer. University of Groningen, University Medical Center Groningen, Groningen, Netherlands

Background: Giant cell arteritis (GCA) and polymyalgia rheumatica (PMR are inflammatory diseases requiring long-term treatment with 